\title{
ADJUSTMENTS OF THE THOMSON BRIDGE IN THE MEASUREMENT OF VERY LOW RESISTANCES
}

\author{
By F. Wenner and E. Weibel
}

In a previous paper ${ }^{1}$ the desirability of making certain adjustments in addition to those necessary for establishing the principal balance of the Thomson bridge is discussed and the procedure followed in the Bureau of Standards for making these adjustments is given. The purpose of this paper is to describe somewhat different procedures which have been found to be better in case the resistances under comparison are extremely low.

Referring to Fig. I, $X$ and $Y$ represent the low resistances under comparison, $A$ and $B$ represent those parts of the resistances of the main ratio arms between $\mathrm{n}$ and $\mathrm{g}$, and between $\mathrm{g}$ and $\mathrm{o} ; \alpha$ and $\beta$ represent those parts of the resistances of the auxiliary ratio arms between $\mathrm{n}^{\prime}$ and $\mathrm{g}^{\prime}$; and between $\mathrm{g}^{\prime}$ and $\mathrm{o}^{\prime}$; and $C$ represents the resistance between $\mathrm{X}$ and $Y$ (approximately the difference in potential between $\mathrm{n}^{\prime}$ and $\mathrm{o}^{\prime}$ divided by the test current). The resistances of the connections between the low resistances (including that of their leads to the potential terminals) and the terminals $\mathrm{n}, \mathrm{n}^{\prime}, \mathrm{o}$, and $\mathrm{o}^{\prime}$ of the double ratio set are represented by $x_{1}, x_{2}, y_{1}$, and $y_{2}$. The branch points to the galvonometer are represented by $g$ and $\mathrm{g}^{\prime}$ and to the battery or other source of current by $b$ and $b^{\prime}$.

In the previous paper it was shown that the equation for the Thomson bridge could be put in the following form:

$$
X=Y A / B[\mathrm{I}+a+D(a-b)]
$$

where $a$ and $D(a-b)$ are small correction terms. More specifically $a$ is the difference (in proportional parts) between the value of the 
main ratio, $\left(A+x_{1}\right) /\left(B+y_{1}\right)$, and $A / B ; a-b$ is the difference (in proportional parts) between the value of the main and auxiliary ratios, and $D$ is approximately equal to the ratio of $C$ to $X+Y$. The purpose of the auxiliary adjustments is to make these correction terms negligibly small and thus save the time which otherwise would be required for determining their magnitudes.

If a switch or other means is provided for opening the low resistance connection between $X$ and $Y$, the bridge can readily be changed from a double (Thomson) to a simple (Wheatstone) bridge and back to a double bridge by simply opening and closing the switch. If, in addition, a means is provided for changing the

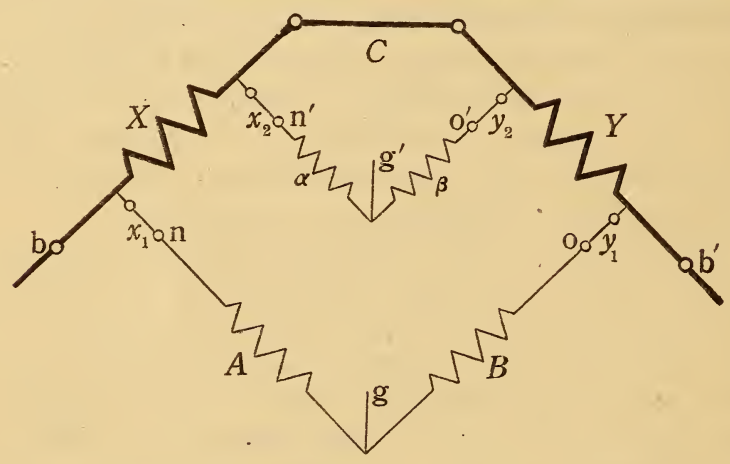

FIG, I

resistances of the auxiliary ratio without changing those of the main ratio, $a-b$ can be made very small, so that if $D$ is considerably less than unity the correction term $D(a-b)$ can be made negligible. This procedure, first published by Reeves, ${ }^{2}$ is now used in most of the precise measurements of low resistances by the Thomson bridge method.

To keep $D$ less than unity-that is, $C$ less than $X+Y$-is difficult if $X$ and $Y$ are both small-o.oooor ohm or less. In such cases the use of a switch is more or less impracticable, on account of its resistance and to open and close the connection at a terminal of one of the resistances one or more times during each measurement requires a considerable amount of extra work. Also the 
time required, in some cases, makes it impossible to follow changes in the resistances caused by rapid changes in their temperature. It therefore seemed advisable to see if a method could be devised for making the adjustments without the repeated opening and closing of the low resistance connection between $X$ and $Y$. On investigation two satisfactory procedures were found. Both require the use of a variable double ratio-set so adjusted that for any setting of the dial-switches the lack of equality of the two ratios $A / B$ and $\alpha / \beta$ is so small that no appreciable error is introduced on this account. The first also requires the use of two

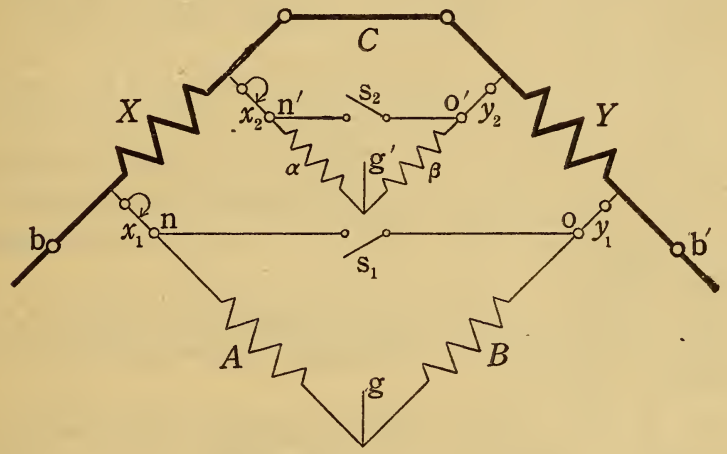

FIG. 2

switches $s_{1}$ and $s_{2}$ (see Fig. 2), and of adjustable low resistances ${ }^{1}$ in the connectors $x_{1}$ and $x_{2}$.

The procedure for the first method then is as follows:

I. With the connections as shown in Fig. $2, s_{1}$ and $s_{2}$ open, the bridge is balanced by an adjustment of the dial switches of the double radio-set.

2. With the switch $s_{1}$ closed $^{3}$ the bridge is balanced by an adjustment of the variable low resistance forming a part of $x_{1}$.

3. With the switch $s_{1}$ open and $s_{2}$ closed, the bridge is balanced by an adjustment of the variable low resistance forming part of $x_{2}$.

4. With the switches $s_{1}$ and $s_{2}$ both open, the bridge is balanced by an adjustment of the dial switches of the double ratio-set. 
The first adjustment makes $A / B$, approximately, equal to $X / Y$; the second makes the correction term $a$ negligibly small, the third makes $b$ very small, and therefore the correction term $D(a-b)$ negligibly small, and the fourth makes

$$
\bar{A}=\frac{X}{\bar{Y}}
$$

If the fourth adjustment requires much change in the reading of the dial switches, the second and third adjustments may be disturbed slightly. In that case the second, third, and fourth adjustments should be repeated, and only the last reading of the dial switches used.

The second procedure requires that, instead of the switches $s_{1}$ and $s_{2}$, means be provided for connecting a battery to the terminals $\mathrm{n}$ and $\mathrm{o}$ and to $\mathrm{n}^{\prime}$ and $\mathrm{o}^{\prime}$. The procedure then is as follows:

I. With the test current supplied through the terminals $b$ and $\mathrm{b}^{\prime}$, the bridge is balanced by an adjustment of the dial switches of the double ratio-set.

2. With the test current supplied through the terminals $n$ and $o$, the bridge is balanced by an adjustment of the variable low resistance forming a part of $x_{1}$.

3. With the test current supplied through the terminals $\mathrm{n}^{\prime}$ and $\mathrm{o}^{\prime}$, the bridge is balanced by an adjustment of the variable low resistance forming a part of $x_{2}$.

4. With the test current supplied through the terminals $b$ and $\mathrm{b}^{\prime}$, the bridge is balanced by an adjustment of the dial switches of the double ratio-set.

Each of these adjustments accomplishes the same purpose as the corresponding adjustment of the first procedure.

Both procedures accomplish the desired result about equally well.

WASHINGTON, May 25, I9I4. 